\title{
Best Estimate Plus Uncertainty Analysis of Departure from Nucleate Boiling Limiting Case with CASL Core Simulator VERA-CS in Response to PWR Main Steam Line Break Event
}

\author{
Cameron S. Brown ${ }^{1}$, Hongbin Zhang ${ }^{2 *}$, Vefa Kucukboyaci ${ }^{3}$, Yixing Sung ${ }^{3}$ \\ ${ }^{1}$ Department of Nuclear Engineering, North Carolina State University \\ 2500 Stinson Drive, Raleigh, NC 27695-7909, USA \\ ${ }^{2}$ Idaho National Laboratory, P.O. Box 1625, Idaho Falls, ID 83415-3870, USA \\ ${ }^{3}$ Westinghouse Electric Company, 1000 Westinghouse Drive, Cranberry Township, PA 16066, USA \\ csbrown3@ncsu.edu; *Hongbin.Zhang@inl.gov; \\ kucukbvn@westinghouse.com; sungy@westinghouse.com
}

\begin{abstract}
VERA-CS (Virtual Environment for Reactor Applications, Core Simulator) is a coupled neutron transport and thermal-hydraulics subchannel code under development by the Consortium for Advanced Simulation of Light Water Reactors (CASL). VERA-CS was applied to simulate core behavior of a typical Westinghouse-designed 4-loop pressurized water reactor (PWR) with 17x17 fuel assemblies in response to two main steam line break (MSLB) accident scenarios initiated at hot zero power (HZP) at the end of the first fuel cycle with the most reactive rod cluster control assembly stuck out of the core. The reactor core boundary conditions at the most DNB limiting time step were determined by a system analysis code. The core inlet flow and temperature distributions were obtained from computational fluid dynamics (CFD) simulations. The two MSLB scenarios consisted of the high and low flow situations, where reactor coolant pumps either continue to operate with offsite power or do not continue to operate since offsite power is unavailable. The best estimate plus uncertainty (BEPU) analysis method was applied using Wilks' nonparametric statistical approach. In this demonstration of BEPU application, 59 full core simulations were performed for each accident scenario to provide the minimum departure from nucleate boiling ratio (MDNBR) at the 95/95 (95\% probability with $95 \%$ confidence level) tolerance limit. A parametric goodness-of-fit approach was also applied to the results to obtain the MDNBR value at the 95/95 tolerance limit. Initial sensitivity analysis was performed with the 59 cases per accident scenario by use of Pearson correlation coefficients. The results show that this typical PWR core retains design margin with respect to the MDNBR safety limit for both of the MSLB accident scenarios. The scenario with available offsite power was more restrictive in terms of MDNBR than the scenario without offsite power.
\end{abstract}

Keywords: Best Estimate Plus Uncertainty, Wilks statistical approach, VERA-CS, Main Steam Line Break, MDNBR

${ }^{*}$ Corresponding author

(C) 2016. This manuscript version is made available under the Elsevier user license http://www.elsevier.com/open-access/userlicense/1.0/ 


\begin{tabular}{ll}
\multicolumn{2}{l}{ ABREVIATIONS } \\
BEPU & Best estimate plus uncertainty \\
CASL & Consortium for Advanced Simulation of Light Water Reactors \\
CC & Correlation coefficient \\
CFD & Computational fluid dynamics \\
CHF & Critical heat flux \\
CTF & COBRA-TF \\
DNB & Departure from nucleate boiling \\
DNBR & Departure from nucleate boiling ratio \\
EOC & End of cycle \\
FOM & Figure of merit \\
HF & High flow \\
HFP & Hot full power \\
HPC & High performance computing \\
HZP & Hot zero power \\
INL & Idaho National Laboratory \\
LF & Low flow \\
LOCA & Loss-of-coolant accident \\
MDNBR & Minimum departure from nucleate boiling ratio \\
MSLB & Main steam line break \\
OECD & Organisation for Economic Co-operation and Development \\
PWR & Pressurized water reactor \\
RCCA & Rod Cluster Control Assembly \\
SA & Sensitivity analysis \\
UQ & Uncertainty quantification \\
VERA-CS & Virtual Environment for Reactor Applications, Core Simulator \\
VUSAT & VERA-CS Uncertainty and Sensitivity Analysis Toolkit \\
WBN1 & Watts Bar Nuclear Unit 1 \\
&
\end{tabular}

\section{INTRODUCTION}

The increasing capabilities of high performance computing (HPC) continue to improve the fidelity of nuclear reactor safety and operational calculations in multi-physics and multi-scales. For such high fidelity calculations, proper uncertainty quantification (UQ) of the computing models of reactor neutronics and thermal-hydraulics is important to provide reliable and accurate results. UQ becomes a necessary step in reactor safety calculations that refers to the determination of uncertainty in model outputs based on the uncertainty in model inputs (Helton, et al. 2006). Uncertainties in safety calculations with computer codes for nuclear reactor modeling and simulation result from code limitations, scaling inaccuracies embedded in the experimental data used for benchmarking, and uncertainties associated with the state of the reactor at the initiation of the transient (Boyack, et al. 1990). UQ seeks to characterize the uncertainties associated with the reactor model and its input values. Specifically, UQ addresses epistemic uncertainty that results from the inability to know the correct value for a model input that is assumed constant (Marcum and Brigantic 2015).

The best estimate plus uncertainty (BEPU) analysis method (Boyack, et al. 1990) has been developed for UQ in support of regulatory rulemaking changes of safety analyses in the nuclear industry (Zhang, et al. 2016) and has been applied to loss-of-coolant accidents (LOCA) (Boyack, et al. 1990) (Frepoli 2007) (Martin and O'Dell 2005) (Perez, et al. 2011). The BEPU method can also be applied to non-LOCA nuclear reactor accident scenarios such as a PWR main steam line break (MSLB) accident. Benchmark simulations for the MSLB scenario with coupled neutronic/thermal-hydraulic considerations have been 
performed under the OECD Nuclear Energy Agency (Ivanov, et al. 1999). During a postulated PWR MSLB event initiated from the hot zero power (HZP) condition, increased steam flow from the broken steam pipe in one of the steam generators results in a significant reduction in the primary coolant temperature and the reactor core is returned to power with a high peak fuel rod power, thus imposing a challenge to the fuel thermal limit with respect to Departure from Nucleate Boiling (DNB). The safety analysis further assumes that the most reactive Rod Cluster Control Assembly (RCCA) is in its fully withdrawn position during the transient. A return to power following a steam line rupture is a potential problem mainly because of the high power peaking factors due to the stuck RCCA assumption. The event is terminated after the boric acid is delivered to the reactor core by the safety injection system. Although it is classified as a Condition IV event that allows fuel cladding failures for radiological dose evaluation, many plant safety analyses conservatively show no fuel rod failure by meeting the DNB Ratio (DNBR) limit with a $95 \%$ probability at a $95 \%$ confidence level (Sherder and McHugh 1998).

The BEPU methodology consists of several sequential and logical steps in the evaluation process. Some of the key steps include: 1) Selection of a plant/core model, accident scenario, safety analysis tools, safety acceptance criteria, and figure of merit (FOM); 2) Selection of relevant physical phenomena and uncertain parameters with their respective probability distribution functions; 3) Construction of accident simulation models; 4) Random sampling of uncertain input parameters and performing accident progression simulations; 5) Determination of the 95/95 (95\% probability with $95 \%$ confidence level) tolerance limits for the FOM. A BEPU analysis can be based on either the Monte Carlo approach or Wilks' nonparametric statistical approach (Wilks 1941). The Monte Carlo approach requires a large number of computer simulations, which presents a challenge when computationally intensive high fidelity and coupled multi-physics codes are used. On the other hand, the Wilks' approach uses a relatively small number of samples to provide the safety metric at the 95/95 tolerance limit. For the Wilks' nonparametric statistical approach, all of the selected uncertain input parameters are sampled simultaneously in the space defined by their respective uncertain ranges (Zhang, et al. 2016). The combined effect of the uncertain parameters on the selected FOM can then be quantified in the safety analysis. When only one FOM is considered, the number of samples required can be determined by:

$$
\beta=1-\gamma^{N}
$$

where $\beta$ is the confidence level, $\mathrm{N}$ is the number of samples, and $\gamma$ is the percentile of probability (Zhang, et al. 2016). For example, using Eq. (1) to determine the $95 / 95$ value of a single FOM, i.e. $\gamma=0.95$ and $\beta$ $=0.95$, would require that the number of samples be 59 .

In this work, a BEPU analysis is demonstrated using the Wilks' nonparametric statistical approach and a parametric approach with goodness-of-fit test to analyze the reactor response for two MSLB accident scenarios. The reactor core responses to the MSLB accident scenarios were simulated using the Virtual Environment for Reactor Applications, Core Simulator (VERA-CS) under development by the Consortium for Advanced Simulation of Light Water Reactors (CASL). Details on VERA-CS are provided in Section 2. The transient response of the reactor coolant system was predicted using a system analysis code RETRAN and the associated plant model for the Westinghouse-designed 4-loop PWR (Huegel, et al. 1999). The reactor core boundary condition consisting of loop flow and temperature, system pressure and core average power at the DNB limiting time step of the transient, referred to as the state point, were determined from the system transient simulation. The core response was analyzed with VERA-CS at the most limiting state point determined by the MSLB accident analysis using RETRAN. In the MSLB accident, the minimum departure from nucleate boiling ratio (MDNBR) is the considered FOM. In general, UQ studies of reactor response require reactor power characteristics (power shape, peaking factors, etc.) as inputs to the thermal-hydraulic code (Marcum and Brigantic 2015). Marcum and Brigantic (2015) found the axial and radial power factors to be the most influential parameters on the minimum departure from nucleate boiling ratio (MDNBR). Predictions of the core thermal response and 
power distributions could be improved with neutronic/thermal-hydraulic coupling. Therefore, it is of interest to use fully-coupled neutronics and core thermal-hydraulic calculations to perform nuclear reactor core uncertainty quantification. VERA-CS provides this fully-coupled neutronic/thermal-hydraulic capability as well as isotopic depletion. Coupled calculations pose a challenge to UQ since, in general, they require more computational power and longer simulation times than stand-alone thermal-hydraulic calculations.

\section{VERA-CS}

The CASL VERA-CS code currently includes three main components: MPACT for reactor physics and neutron transport, COBRA-TF for thermal-hydraulics, and ORIGEN for isotopic depletion (Collins and Godfrey 2015). VERA-CS has been tested and applied to an array of problems including core physics analysis (Franceshini, et al. 2015), full-core modeling for all of the fuel cycles of Watts Bar Nuclear Unit 1 (WBN1) (Kochunas, et al. 2015) (Godfrey, et al. 2015), and startup core modeling for the AP1000 PWR (Franceshini, et al. 2015).

MPACT is a 3D pin-resolved reactor transport code developed by the Oak Ridge National Laboratory (ORNL) and the University of Michigan. MPACT uses the 2D/1D method to solve the neutron flux distribution throughout the specified geometry. In the 2D/1D method, the 2D method of characteristics (MOC) is used in the radial planes in order to capture the heterogeneity in the radial direction with high accuracy; where each pin cell is explicitly modeled and even sub-pin detail can be captured (Collins and Godfrey 2015). In the axial direction a low-order transport solution using $\mathrm{SP}_{3}$ is obtained through a pincell homogenized basis (Collins and Godfrey 2015). Axial and radial solutions are linked through the use of transverse leakage terms that ensure neutron balance in every pin-cell at convergence (Collins and Godfrey 2015). MPACT uses a 47 energy group library based on ENDF/B VII.1 data with subgroup parameters to capture self-shielding effects (Collins and Godfrey 2015). More details on MPACT methodology can be found in Collins and Godfrey (2015), Kochunas et al. (2015), and Collins et al. (2014).

COBRA-TF (CTF) is a multi-phase/fluid T/H code with improvements for subchannel analysis made by ORNL and Pennsylvania State University (Salko, et al. 2015). CTF solves the mass, momentum, and energy equations for the liquid, vapor and droplet phases for each subchannel in the core, and predicts the axial flow and enthalpy distributions within each channel as well as cross-flow and lateral energy exchanges between channels. Direct coupling between neutronics (MPACT) and thermal-hydraulics (CTF) in VERA-CS provides coolant/fuel temperatures and density feedbacks from CTF to MPACT to iterate on the power distribution. CTF includes a number of models important for LWR safety and design analysis including flow regime dependent two-phase wall heat transfer, inter-phase heat transfer and drag, droplet breakup, and quench-front tracking (Kochunas, et al. 2015). A more in-depth discussion on the coupling between MPACT and CTF can be found in Kochunas, et al. (2015). CTF was evaluated for the MSLB application in Y. Sung, et al. (2015a).

ORIGEN (Gauld, et al. 2011) is developed by ORNL and released through the SCALE package. ORIGEN is directly integrated in MPACT through its API to calculate the changes in nuclides for every depletable cross-section region (Collins and Godfrey 2015). A reduced isotope library and number of isotopes have been used for this study. 


\section{BEPU METHODOLOGY WITH VERA-CS}

\subsection{Problem Description and Model Framework}

Recently, work performed under the CASL program (Y. Sung, et al. 2015b) (Kucukboyaci, et al. 2016) used VERA-CS to assess the core response to a MSLB event that poses a challenge to the departure from nucleate boiling (DNB) criteria. Since the minimum DNB ratio (MDNBR) occurs minutes after initiation of the transient, the reactor core response at the MDNBR time step can be simulated in a quasi-steadystate mode similar to the current industrial practice (Sherder and McHugh 1998). In addition to the MSLB, the most reactive rod cluster control assembly (RCCA) is assumed stuck out of the core to exacerbate the accident conditions. In order to capture the asymmetric power distribution in the reactor core due to the broken steam pipe in one loop and the stuck RCCA, the VERA-CS model was set up for the whole reactor core. Reactor state points for this scenario were obtained through use of a system analysis code for the "high flow" (HF) situation where offsite power was available and the reactor coolant pumps continued to operate, as well as the "low flow" (LF) situation where offsite power was unavailable and the reactor coolant pumps did not continue to operate. These reactor state points were used as boundary conditions in the current work to perform high fidelity reactor core response simulations with VERA-CS for both the high flow and low flow scenarios. The previous work under the CASL program (Y. Sung, et al. 2015a) (Kucukboyaci, et al. 2016) considered one HF and one LF scenario with estimated core inlet distributions. Based on the previous work, the current BEPU analysis validates the conclusion of the DNB limiting case by accounting for uncertainties in the core boundary conditions and core modeling parameters and assessing their effects on the FOM. The typical PWR core design parameters at hot full power (HFP) conditions are shown in Table 1.

Table 1. Typical PWR core design parameters at hot full power conditions used in VERA-CS.

\begin{tabular}{|c|c|c|}
\hline Parameter & Value & Unit \\
\hline Core Power & 3411 & $\mathrm{MW}_{\text {th }}$ \\
\hline Inlet Temperature & $557.7(292)$ & ${ }^{\circ} \mathrm{F}\left({ }^{\circ} \mathrm{C}\right)$ \\
\hline Inlet Flow Rate & $2.32(3147)$ & $\mathrm{Mlbm} / \mathrm{hr}-\mathrm{ft}^{2}\left(\mathrm{~kg} / \mathrm{m}^{2}-\mathrm{s}\right)$ \\
\hline System Pressure & $2250(15.5)$ & $\mathrm{psia}(\mathrm{MPa})$ \\
\hline
\end{tabular}

The reactor model uses typical 17x17 PWR fuel assemblies with 264 fuel rods, 24 guide tubes, and 1 instrumentation tube (Figure 1). VERA-CS takes into account core and fuel assembly properties such as the upper and lower core plates, lower and upper assembly nozzles, and guide/instrument tubes. Eight spacer grids are included in the assemblies to provide structural support as well as improve coolant mixing. Parameters for the 17x17 PWR fuel assemblies used in this work are shown in Table 2. Assemblies with fuel enrichments of $2.110 \%, 2.619 \%$, and $3.100 \% \mathrm{U}^{235}$ were specified at core locations shown in Figure 2. A fuel burnup calculation was first performed with VERA-CS for cycle 1 of this PWR setup using the HFP conditions of Table 1, much like in Kochunas, et al. (2015), to create a restart file at EOC when the MSLB is initiated. 
Table 2. Typical 17x17 PWR fuel assembly parameters used in VERA-CS.

\begin{tabular}{|c|c|c|}
\hline Parameter & Value & Unit \\
\hline Fuel Enrichment & $2.110 / 2.619 / 3.100$ & $\mathrm{~cm}$ \\
\hline Active Core Length & 365.76 & $\mathrm{~cm}$ \\
\hline Rod Pitch & 1.26 & - \\
\hline Clad Material & ZIRC & $\mathrm{cm}$ \\
\hline Clad Outer Radius & 0.475 & $\mathrm{~cm}$ \\
\hline Clad Inner Radius & 0.418 & $\mathrm{~cm}$ \\
\hline Fuel Material & $\mathrm{UO}_{2}$ & - \\
\hline Fuel Pellet Outer Radius & 0.4096 & $\mathrm{~cm}$ \\
\hline Instrument Tube Material & ZIRC & $\mathrm{cm}$ \\
\hline Instrument Tube Thickness & 0.041 & $\mathrm{~cm}$ \\
\hline Instrument Tube Outer Radius & 0.602 & 21.5 \\
\hline Assembly Pitch & & \\
\hline
\end{tabular}

Due to the MSLB occurring in one of the reactor plant loops, the core inlet temperature and mass flux distributions are not uniform. Commercially available computational fluid dynamics (CFD) software, STAR-CCM+10.04.01, was applied to predict the core inlet temperature and mass flux distributions as input to VERA-CS simulation of the reactor core response at the DNB limiting time step of the MSLB accident. The CFD model included cold legs, downcomer annular, lower plenum, lower core support plate, core plate, and a plenum of porous media above the core plate to simulate the reactor core. Flow turbulence was modeled using the two equation k- $\omega$ SST model with curvature corrections. The CFD modeling accounted for buoyancy force and gravitational effect. The reactor core inlet temperature and mass flux distributions on an assembly-by-assembly basis from the CFD simulations as input to VERACS for the high and low flow cases are shown in Figure 3 through Figure 6. A VERA-CS sensitivity study (Kucukboyaci, et al. 2016) concluded that the DNBR responses under the MSLB conditions were insensitive to uncertainties in the inlet temperature and flow distributions from the CFD predictions. VERA-CS has the capability to define inlet temperature (Figure 3 and Figure 4) and mass flux (Figure 5 and Figure 6) distributions on an assembly-by-assembly basis and that capability was used in the analysis. Figure 3 and Figure 4 show the core inlet temperature distribution as the difference in degrees Fahrenheit from the average value (shown in Table 3) for the HF and LF scenarios, respectively. Figure 5 and Figure 6 show the inlet mass flow rate fraction distribution that is multiplied by the average inlet mass flow rate specified in VERA-CS for the HF and LF scenarios, respectively.

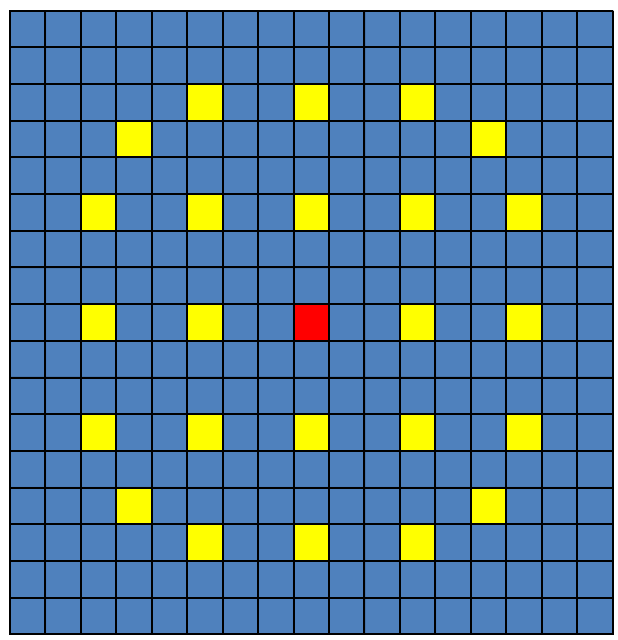

Figure 1. Typical PWR 17x17 fuel assembly. The yellow cells represent locations of guide tubes, the red cell indicates the instrumentation tube (center of assembly), and blue cells represent fuel pin locations. 
Figure 7 shows the full core RCCA map and assemblies where no RCCA is present are denoted by a hyphen. The most reactive RCCA is stuck out of the core and is shown by the location in red for both the HF and LF scenarios. All other RCCAs are fully inserted into the core for the MSLB accident scenarios considered in this work.

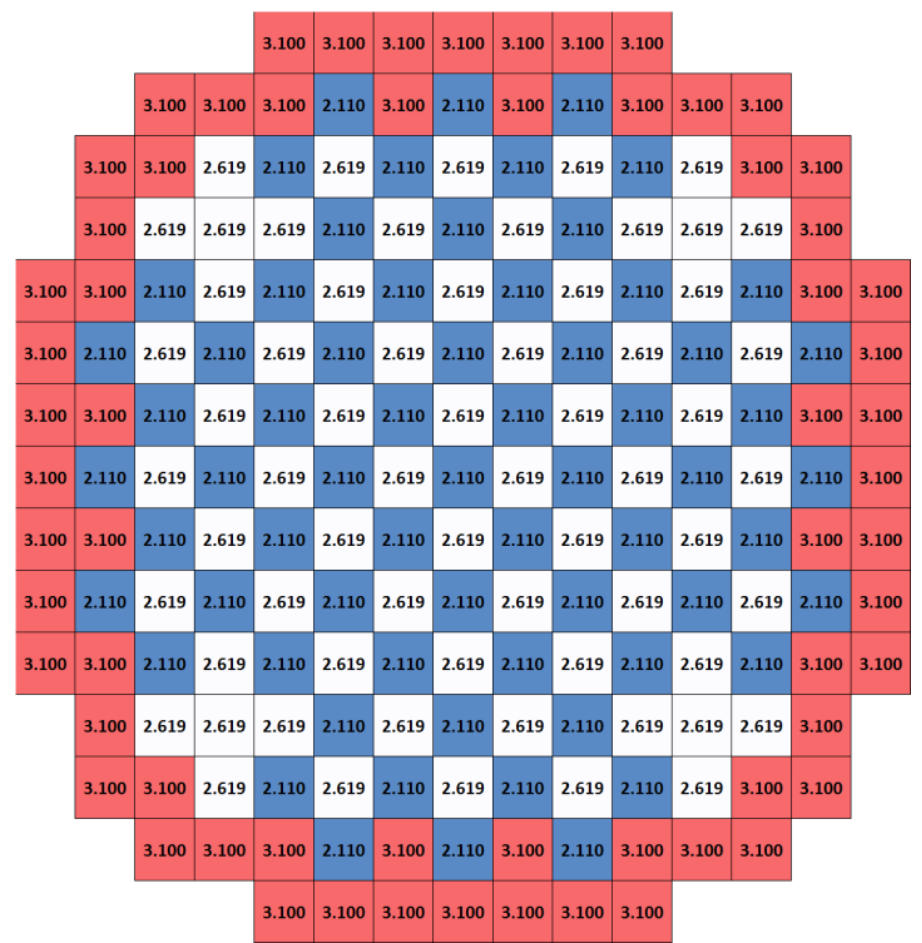

Figure 2. Full core loading pattern of assemblies enriched to $2.110 \%, 2.619 \%$, and $3.100 \% \mathrm{U}^{235}$.

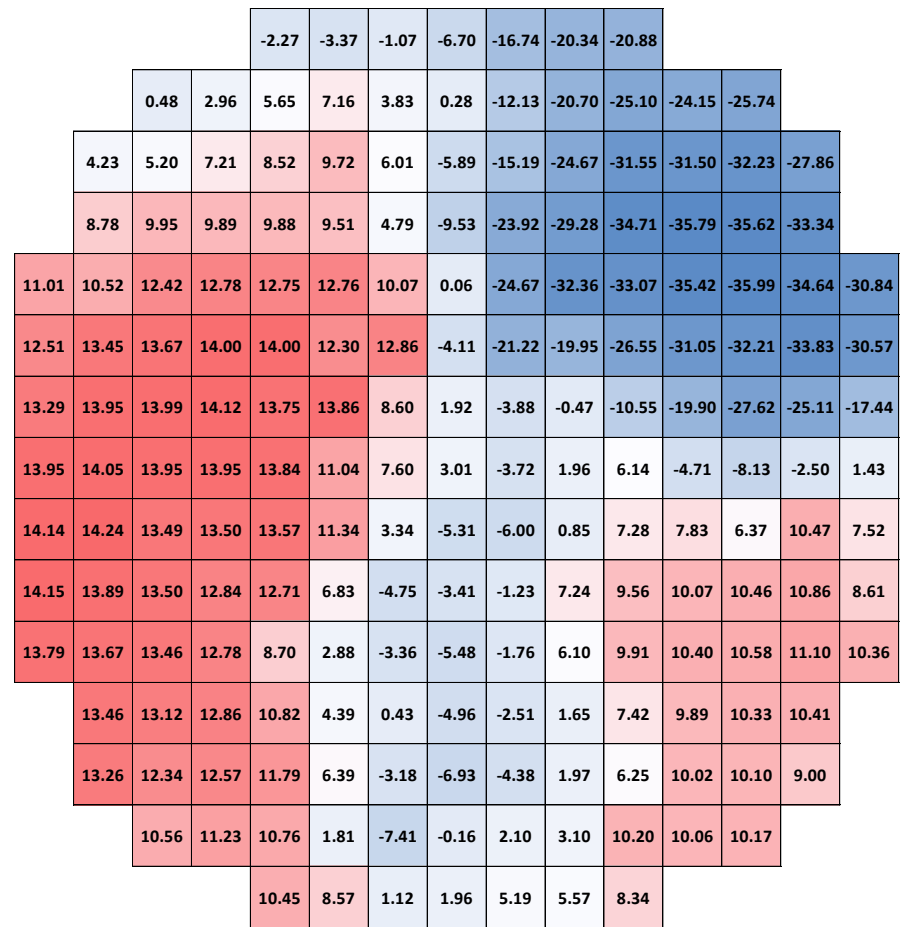

Figure 3. HF scenario core inlet temperature distribution $\left({ }^{\circ} \mathrm{F}\right)$ on a per-assembly basis as the difference from the average value. 


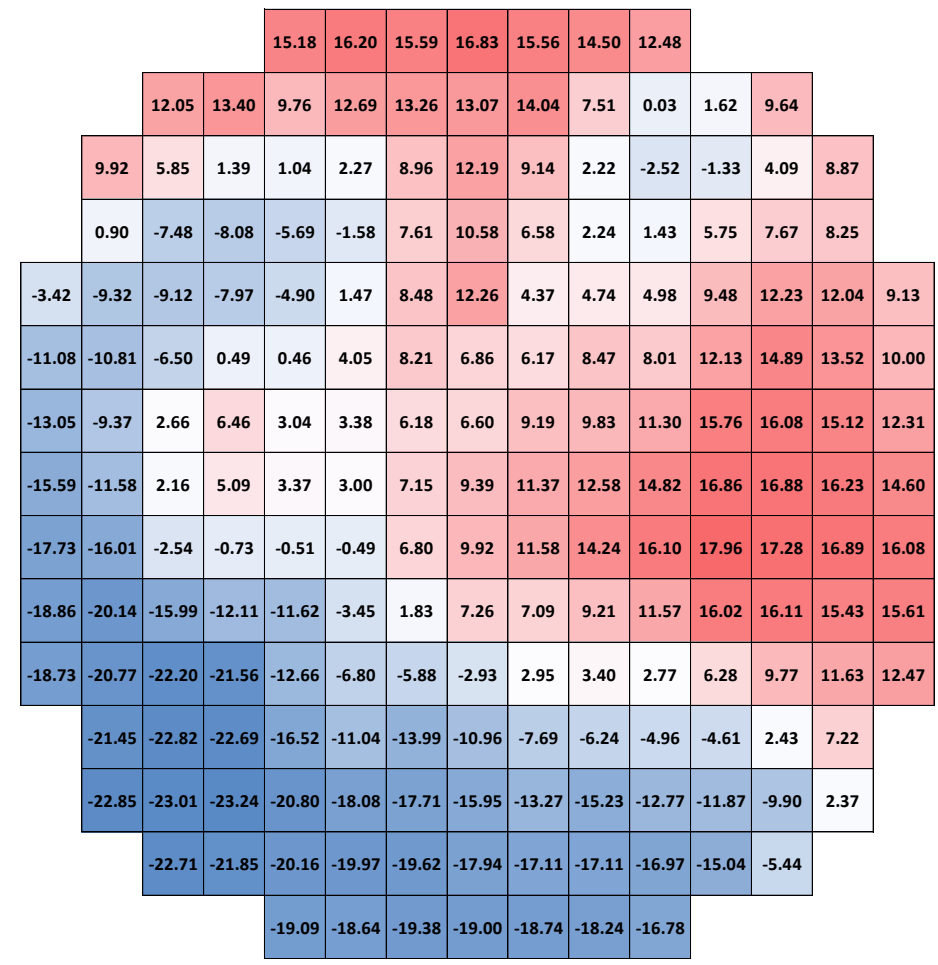

Figure 4. LF scenario core inlet temperature distribution $\left({ }^{\circ} \mathrm{F}\right)$ on a per-assembly basis as the difference from the average value.

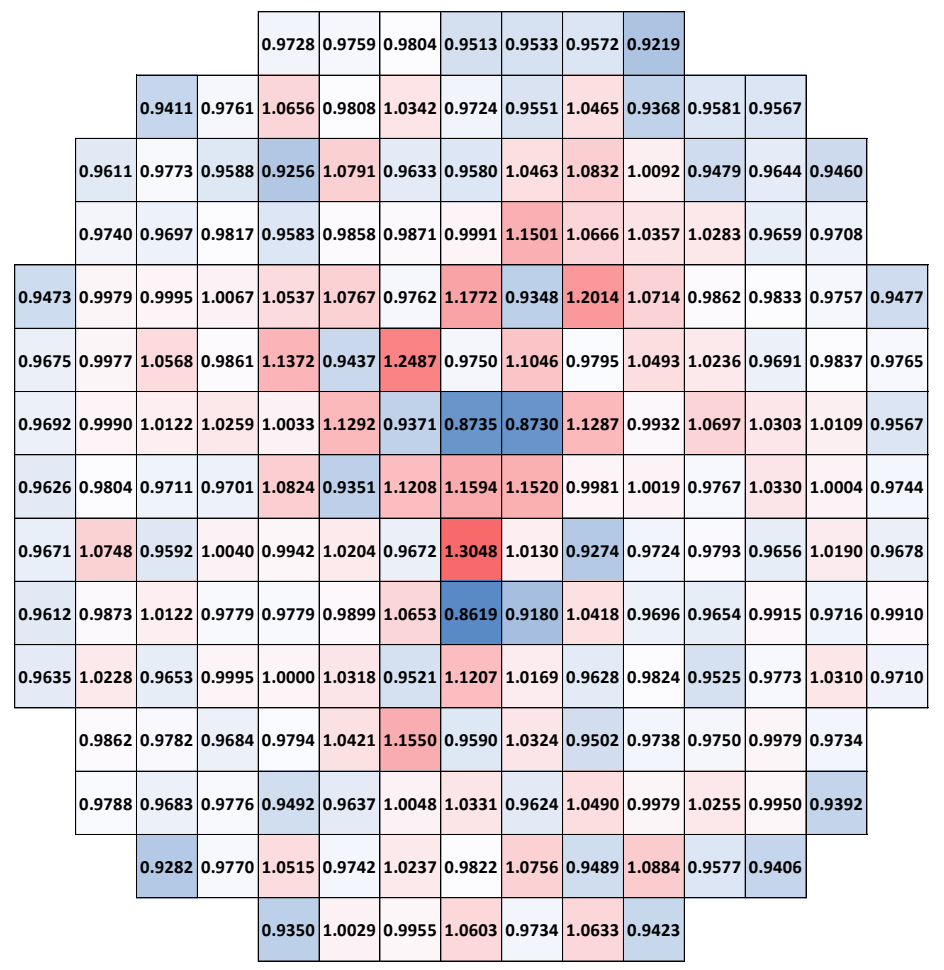

Figure 5. HF scenario core inlet mass flow rate distribution on a per-assembly basis as a fraction of the average value. 


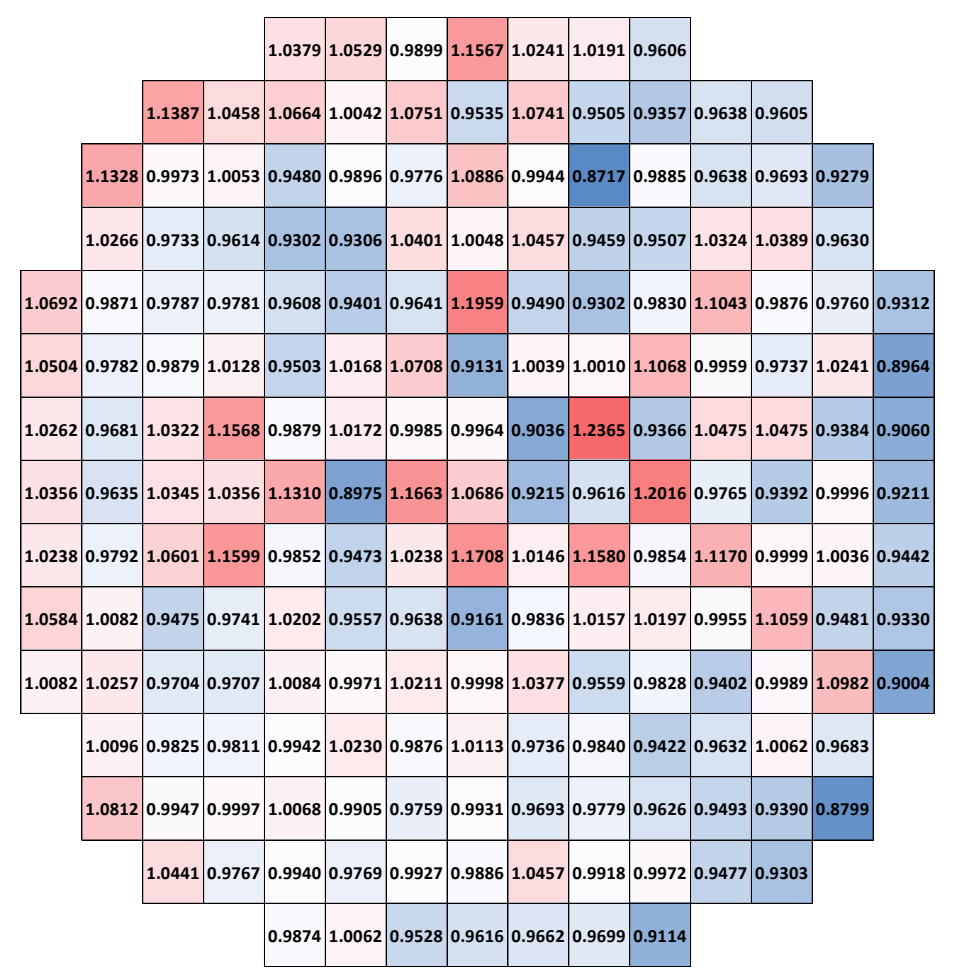

Figure 6. LF scenario core inlet mass flow rate distribution on a per-assembly basis as a fraction of the average value.

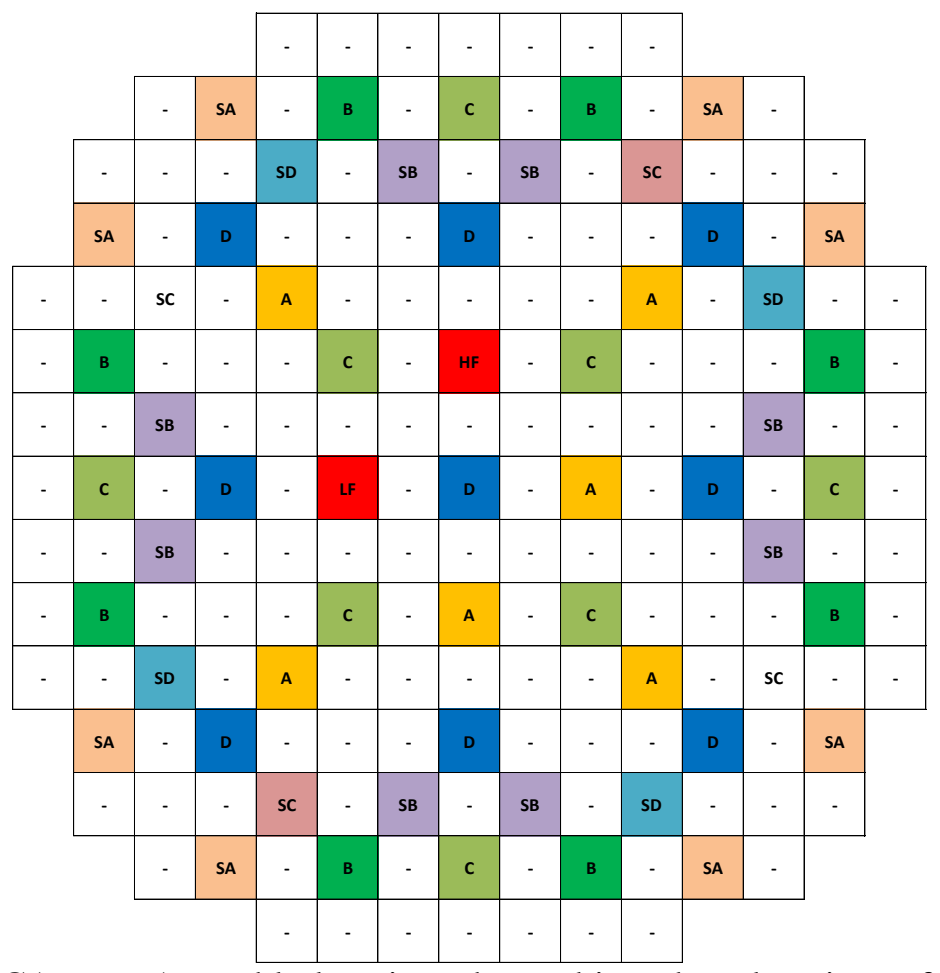

Figure 7. Full core RCCA map. Assembly locations denoted in red are locations of the withdrawn RCCA in the labeled HF and LF scenarios. No RCCA is present in assembly locations denoted with a hyphen. 


\subsection{Figure of Merit - The Minimum Departure from Nucleate Boiling Ratio}

Performing uncertainty quantification requires that a relevant system response output variable, or figure of merit (FOM), be chosen to analyze. VERA-CS provides a number of system response variables that could be analyzed and in this work the MDNBR is chosen as the FOM. The MDNBR is the minimum departure from nucleate boiling ratio calculated anywhere within the reactor core:

$$
D N B R=\frac{q_{C H F}^{\prime \prime}}{q_{\text {local }}^{\prime \prime}} \quad M D N B R=\min (D N B R)
$$

where $q_{\text {local }}^{\prime \prime}$ is the local wall heat flux and $q_{C H F}^{\prime \prime}$ is the critical wall heat flux. In reactor design applications, sufficient margin is maintained to ensure that the local heat flux through the heated wall to the coolant does not reach the critical heat flux value. Therefore, it is of interest in reactor performance analysis codes to calculate the MDNBR everywhere within the reactor core as a safety metric and insure that this value remains above a predetermined safety standard. The safety standard for the PWR MSLB analysis is that the MDNBR is above the limit on a 95/95 basis.

A CTF code option was selected to calculate the critical heat flux value at each node using the W-3 correlation. The W-3 correlation was developed by Tong (Tong 1967) and calculates the critical heat flux as a function of local equilibrium quality, inlet subcooling, pressure, and coolant mass flux. In this study, DNBR values from the HF and LF cases were not only evaluated with respect to the DNBR limit, but were also compared in order to determine the limiting case with respect to DNBR margin to the limit.

\subsection{Identifying Relevant Uncertain Input Parameters}

Input parameters considered in the MSLB accident scenario and their associated uncertainty ranges for the BEPU analysis are shown in Table 3. The input parameters consisted of the reactor core operating parameters and modeling parameters. The uncertainty ranges were representative of those applied in PWR safety analyses. Most of the uncertainty distributions were assumed to be uniform for this evaluation. Also, all uncertainty distributions were assumed to be independent, even though some parameter variations were known to be dependent and correlated such as power and temperature. The assumptions of the uniform and independent distributions tend to lead to larger variations in the DNBR response and to affect margin to the limit, but are not expected to affect the determination of the DNB limiting case between the two cases with the same uncertainties. These parameters were chosen as those that are expected to influence the MDNBR in the core response of a MSLB scenario as well as those relevant parameters that have high input uncertainty and can be varied in the VERA-CS input file. It is intuitive to include the inlet coolant temperature, system pressure, and inlet coolant mass flux from the system transient simulation as sources of uncertainty since the W-3 correlation (Tong 1967) is a function of these variables. Similar values for these parameters were used by Marcum and Brigantic (2015) and Ikonen (2016). In general, studies of reactor response (Marcum and Brigantic 2015) (Frepoli 2007) (Martin and O'Dell 2005) must include core power parameters (e.g. power distribution and peaking factors) as inputs to the thermal-hydraulic code as sources of uncertainty. Although there is inherent uncertainty in the neutronic calculations, the effect of perturbed variables on the core power parameters is not captured in stand-alone thermal-hydraulic codes such as those performed by Marcum and Brigantic (2015). However, the fully-coupled neutronic and thermal-hydraulic capabilities of VERA-CS eliminate this major source of input uncertainty since the core power distribution is calculated directly and thermalhydraulic feedback is considered. This is an important step in reducing uncertainty in reactor safety and operation calculations since the effect of perturbed variables on the power shape is captured by direct calculation. The reactor operating power is an important parameter that has some uncertainty range in MSLB scenarios and plays a major role in the calculation of the linear heat rate and rod heat flux. It is 
therefore expected to influence the MDNBR. The $2 \%$ uncertainty range in core power is applied to the full core rated power and can therefore range from $23.3 \%$ to $27.3 \%$ for the HF scenario and from $9.9 \%$ to $13.9 \%$ for the LF scenario of the $3411 \mathrm{MW}_{\text {th }}$ rating. Input values of some parameters in the VERA-CS model, such as the stuck RCCA rod worth and its location, were chosen in the conservative directions within the uncertainty ranges, in order to be consistent with the modeling requirements for a plant safety analysis. Therefore, those input parameters were excluded from the list in Table 3.

Table 3. Input parameters and their associated uncertainty range considered in the MSLB analysis.

\begin{tabular}{|c|c|c|c|c|}
\hline \multirow{2}{*}{ Model Input Parameter } & \multicolumn{2}{|c|}{ Base Value } & \multirow{2}{*}{$\begin{array}{c}\text { Uncertainty } \\
\text { Range }\end{array}$} & \multirow{2}{*}{ Distribution } \\
\hline & HF & $\mathbf{L F}$ & & \\
\hline Core power & $25.3 \%$ of rated & $11.9 \%$ of rated & $\pm 2 \%$ of rated & Uniform \\
\hline Inlet coolant temperature & $\begin{array}{c}429.4^{\circ} \mathrm{F} \\
\left(220.78^{\circ} \mathrm{C}\right)\end{array}$ & $\begin{array}{c}411.4^{\circ} \mathrm{F} \\
\left(210.78^{\circ} \mathrm{C}\right)\end{array}$ & $\begin{array}{c} \pm 5^{\circ} \mathrm{F} \\
\left( \pm 2.78^{\circ} \mathrm{C}\right)\end{array}$ & Normal \\
\hline System pressure & $\begin{array}{c}518.49 \mathrm{psia} \\
(3.5749 \mathrm{MPa})\end{array}$ & $\begin{array}{c}853.12 \mathrm{psia} \\
(5.8821 \mathrm{MPa}) \\
\end{array}$ & $\begin{array}{c} \pm 20 \mathrm{psia} \\
( \pm 1379 \mathrm{MPa})\end{array}$ & Normal \\
\hline Inlet coolant mass flow rate & $\begin{array}{l}163.87 \mathrm{Mlbs} / \mathrm{hr} \\
(74.330 \mathrm{Mkg} / \mathrm{hr})\end{array}$ & $\begin{array}{c}14.965 \mathrm{Mlbs} / \mathrm{hr} \\
(6.78801 \mathrm{Mkg} / \mathrm{hr})\end{array}$ & $\pm 2 \%$ & Normal \\
\hline $\begin{array}{l}K_{m} \text { : distribution weighting } \\
\text { factor for the CTF void-drift } \\
\text { model }\end{array}$ & \multicolumn{2}{|c|}{1.4} & $\pm 10 \%$ & Uniform \\
\hline $\begin{array}{c}\beta_{\text {sp }}: \text { CTF constant turbulent } \\
\text { mixing coefficient }\end{array}$ & \multicolumn{2}{|c|}{0.005} & $\pm 10 \%$ & Uniform \\
\hline $\begin{array}{l}\text { dhfrac: Fraction of power } \\
\text { deposited directly in coolant }\end{array}$ & \multicolumn{2}{|c|}{0.02} & $\pm 10 \%$ & Uniform \\
\hline Clad outer/inner radius & \multicolumn{2}{|c|}{$0.475 / 0.418$} & $\pm 0.002 \mathrm{~cm}$ & Uniform \\
\hline $\begin{array}{c}\text { Fuel pellet radius } \pm \\
\text { manufacturing tolerance }\end{array}$ & \multicolumn{2}{|c|}{$0.4096 \pm 0.001 \mathrm{~cm}$} & $\begin{array}{r}+0.003 \sim \\
+0.004 \mathrm{~cm} \\
\end{array}$ & Uniform \\
\hline Fuel $\%$ of theoretical density & \multicolumn{2}{|c|}{$94.5 \%$} & $\pm 1.6 \%$ & Uniform \\
\hline Fuel enrichment (\%) & \multicolumn{2}{|c|}{$2.11 / 2.619 / 3.10 \%$} & $\pm 0.05 \%$ & Uniform \\
\hline $\begin{array}{c}\text { Grid spacer form loss - } \\
\text { END/MID }\end{array}$ & \multicolumn{2}{|c|}{$0.9070 / 0.9065$} & $\pm 20 \%$ & Uniform \\
\hline $\begin{array}{l}\text { Pellet-to-clad gap heat transfer } \\
\text { coefficient }\end{array}$ & \multicolumn{2}{|c|}{ 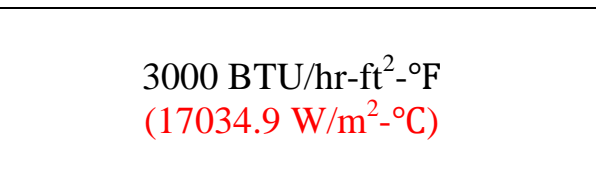 } & $\begin{array}{c} \pm 2000 \\
\mathrm{BTU} / \mathrm{hr}-\mathrm{ft}^{2}-{ }^{\circ} \mathrm{F} \\
( \pm 11356.6 \\
\left.\mathrm{W} / \mathrm{m}^{2}-{ }^{\circ} \mathrm{C}\right)\end{array}$ & Uniform \\
\hline
\end{tabular}

The void-drift model coefficient $\left(K_{m}\right)$, turbulent mixing coefficient $\left(\beta_{\mathrm{sp}}\right)$, and spacer grid form losses are all based on best-estimate models and included as source/sink terms in the CTF momentum equations. Therefore, each of these terms is prescribed a large uncertainty range since the effects of such empirical correlations on calculations should be assessed (Boyack, et al. 1990). For the spacer grid form losses, END denotes those spacer grids at the top and bottom of the assembly and MID refers to the remaining six spacer grids spaced throughout the internal portion of the assembly. The spacer grid form loss inputs are correlated such that the same perturbation percentage is applied to both the END and MID distinctions. The same uncertainty range for the spacer grid form losses were used in previous nuclear thermal-hydraulic UQ/SA studies by Marcum and Brigantic (2015). Much like the models used for source/sink terms in the CTF momentum equations, the fraction of power deposited directly in the coolant (dhfrac) term represents a source/sink term in the CTF energy equations and a sizable uncertainty range is applied to this input as well. Parameters pertaining to the fuel and cladding specifications were chosen based on fuel manufacturing and operational characteristics. In this VERA-CS case, the manufacturing 
tolerances are applied to fuel and cladding specifications (identical or similar tolerances were used in Ikonen (2016)). Due to fuel swelling after cycle 1 operation however, the fuel radius is then perturbed by the uncertainty range in Table 3 . For the inner and outer clad radii the inputs are correlated such that the same perturbation of the manufacturing tolerance is applied to both. The fuel pellet radius and clad inner/outer diameter perturbation was the same for each assembly, regardless of enrichment. The fuel density uncertainty specified here is that from previous sensitivity analysis studies of fuel performance by Ikonen (2016). The pellet-to-clad gap heat transfer coefficient is prescribed a large uncertainty range since the value can vary largely as a function of burnup after cycle 1 operation.

Each of the parameters in Table 3 was sampled from a uniform or truncated normal distribution within the defined uncertainty range. The uniform distribution is considered conservative since maximum and minimum values are equally as likely to occur as the base value (Marcum and Brigantic 2015). Section 3.4 outlines the overall analysis method for performing UQ with VERA-CS.

\subsection{Random Sampling of the Uncertain Parameters using the VUSAT}

To perform and streamline the VERA-CS UQ for the MSLB analysis, the VERA-CS Uncertainty and Sensitivity Analysis Toolkit (VUSAT) was formulated and developed (Brown and Zhang 2016). VUSAT contains two main portions and uses Python scripting and Fortran 95 coding to prepare perturbed VERACS input files and post-process the results for desired information on FOMs (Figure 8).

In Figure 8 the solid lines indicate the first VUSAT portion of pre-processing and VERA-CS execution. This pre-processing step uses a Python script to perform the overall perturbation of the base values. A VERA-CS input file was first created for the base MSLB case with the nominal input values shown in Table 3. The table of uncertain parameters was also created as an input to the Python script. The Python script is then called to read the nominal VERA-CS input file, sample the variables defined in the table of uncertain parameters from the ranges defined in the table of uncertain parameters input file, and then create $N$ new VERA-CS input files with perturbed parameters. In this work, 59 full core MSLB simulations were performed for both the HF and LF scenarios. Each of these input files was placed into a new directory in the Linux file system so that VERA-CS code output for each case was kept separate. A shell script was then used to submit all of the new perturbed cases to the Idaho National Laboratory (INL) HPC queue.

For each of the 59 simulations a number of CTF output files were created for all of the assemblies, namely, a summary file of DNB parameters (i.e. heat flux, CHF, DNBR), an output file with results (e.g. equilibrium quality, mass flux, void fraction, etc.) for each channel within the assembly, and an output file of temperature information for each rod within the assembly. The post-processing portion of the VUSAT (dashed lines in Figure 8) utilized both Python scripting and Fortran 95 coding to first search the DNB output files and find the MDNBR value and location. Data from the channel where the MDNBR occurred were also written to an output file. The final step of the VUSAT retrieved the FOMs from each of the case summary files as well as the values for each of the perturbed parameters defined by the table of uncertain parameter input file. A FOM summary file is then written that contains the FOMs and perturbed values for each case. The same steps encompassed by VUSAT to perform 59 simulations for UQ of the MSLB accident were used for both the HF and LF scenarios. 


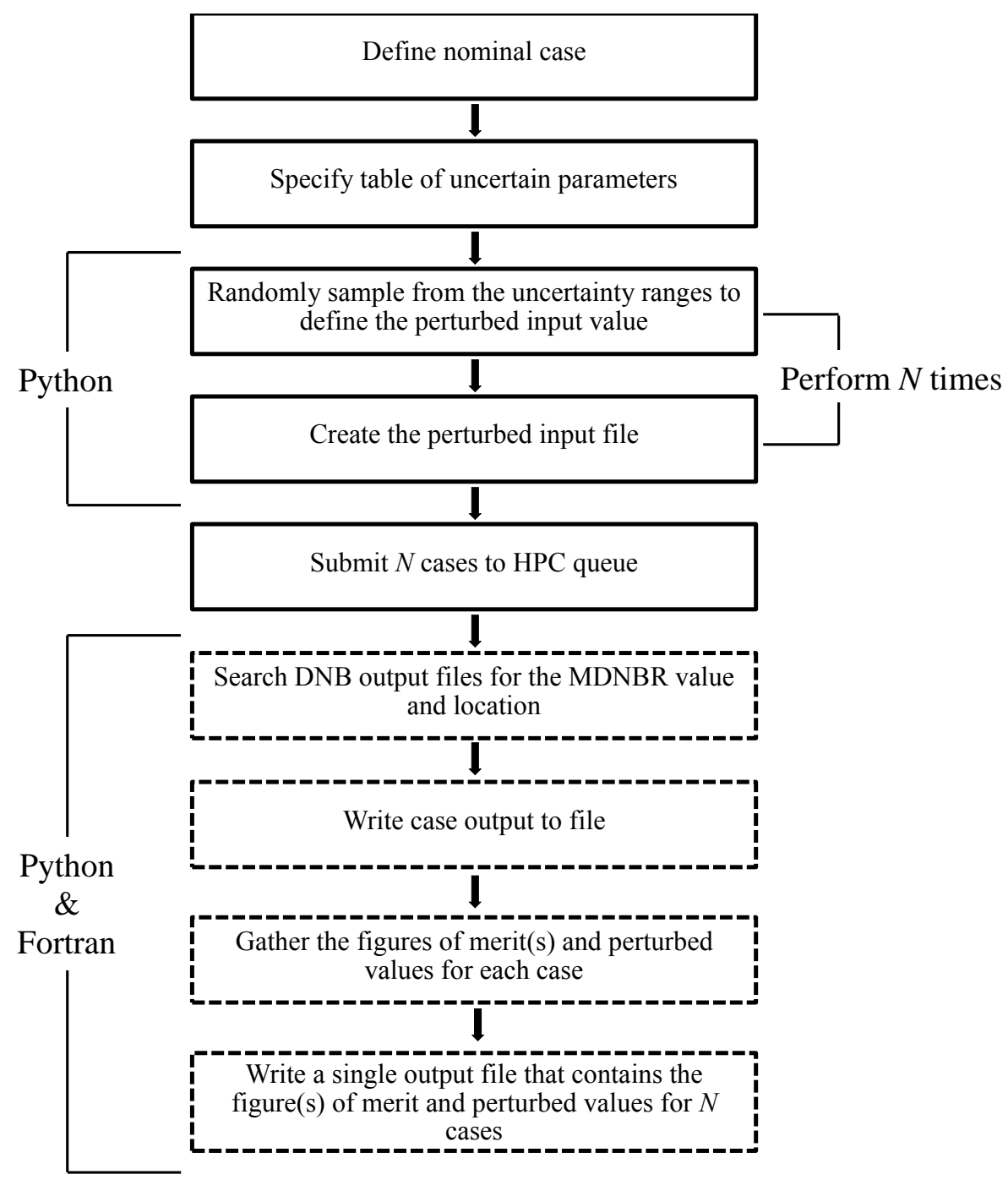

Figure 8. VUSAT summary. Solid lines indicate pre-processing and VERA-CS execution steps while dashed lines indicate post-processing steps.

\subsection{High Performance Computing with VERA-CS}

VERA-CS is a highly scalable code built to run on HPC platforms since fully-coupled neutronic and thermal-hydraulic calculations require considerable computational overhead. This is especially true when considering the entire core geometry rather than simulations in quarter core or one-eighth core geometry. For this work, each one of the 118 simulations was performed on 4408 cores of the Falcon machine at the INL High Performance Computing Center. Falcon is a 19,200 core SGI ICE X distributed memory system with 100 TB of total memory running the Linux operating system. Falcon has benchmarked at 570 TFlops with LINPACK.

The latest version of VERA-CS provides the ability for CTF simulations to be performed on four processors per assembly in the model and that capability is used in the current work. The computation times for both the HF and LF scenarios are shown in Table 4 for the base cases as well as the maximum and minimum computation times. Overall, the perturbation of inputs is not the only contributor to the total simulation time for each case since other factors (e.g. total machine load, different convergence behavior) can have an effect as well. The average simulation times are reasonable to perform UQ with 
Wilks' approach for full core, fully-coupled nuclear reactor analysis but are not affordable for a Monte Carlo approach. The total simulation time for all 118 cases was slightly over one month due to limits on the availability of the Falcon machine (note each VERA-CS simulation required about 23\% of Falcon's computational cores).

Table 4. Computation times for the HF and LF cases.

\begin{tabular}{|c|c|c|}
\hline Computation Time (h) & High Flow & Low Flow \\
\hline Base case & 2.97 & 5.30 \\
\hline Maximum & 8.44 & 8.61 \\
\hline Minimum & 2.63 & 4.48 \\
\hline Average & 3.48 & 4.74 \\
\hline
\end{tabular}

\section{RESULTS}

\subsection{High Flow Scenario}

The HF scenario VERA-CS model results for DNBR and heat flux as functions of distance from the core inlet are shown in Figure 9 for the most limiting, base, and the least limiting cases in the MDNBR channel. For all of these cases, the MDNBR occurred in the assembly with the stuck RCCA. Figure 9 shows how DNBR behaves as an inverse function of heat flux as well as that the axial power shape was peaked in the lower region of the core for this accident scenario. The effects of spacer grids are seen as areas of depression in the heat flux for each case. For each of the cases, the MDNBR occurred at an axial height around 0.35 meters. In Figure 10, the effects of spacer grids are seen as areas of sharp decrease in the total mass flow rate for the channel where the MDNBR occurred. Changes in mass flow rate occur within the hot channel due to cross flow effects between subchannels. However, total mass conservation is observed when considering the entire reactor core. Figure 11 shows the equilibrium quality for the most limiting, base, and least limiting cases. The equilibrium quality is a measure of coolant subcooling (negative equilibrium quality) or superheat (positive equilibrium quality) and Figure 11 shows that each of the cases experiences voiding towards the channel exit. The vapor volume fractions at the channel exit were $44.8 \%, 40.9 \%$, and $32.7 \%$ for the most limiting, base, and least limiting cases, respectively.

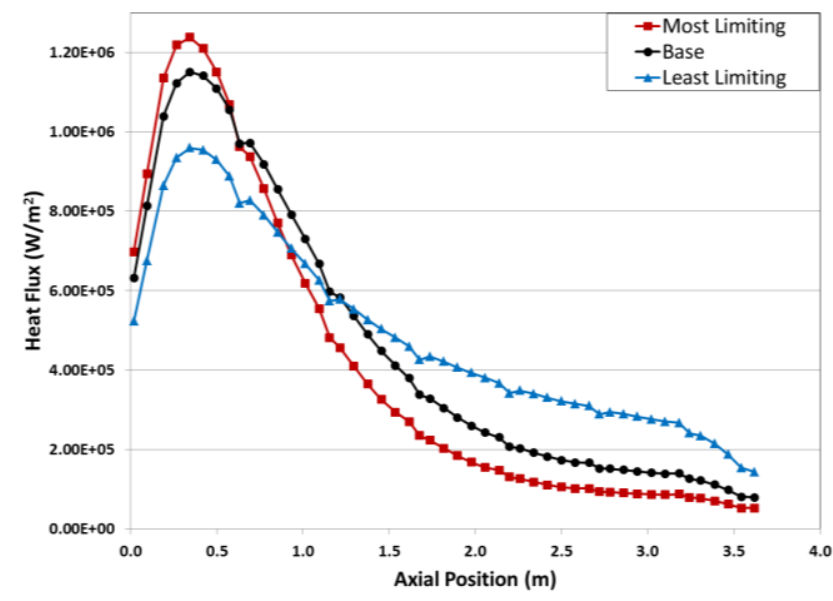

(a)

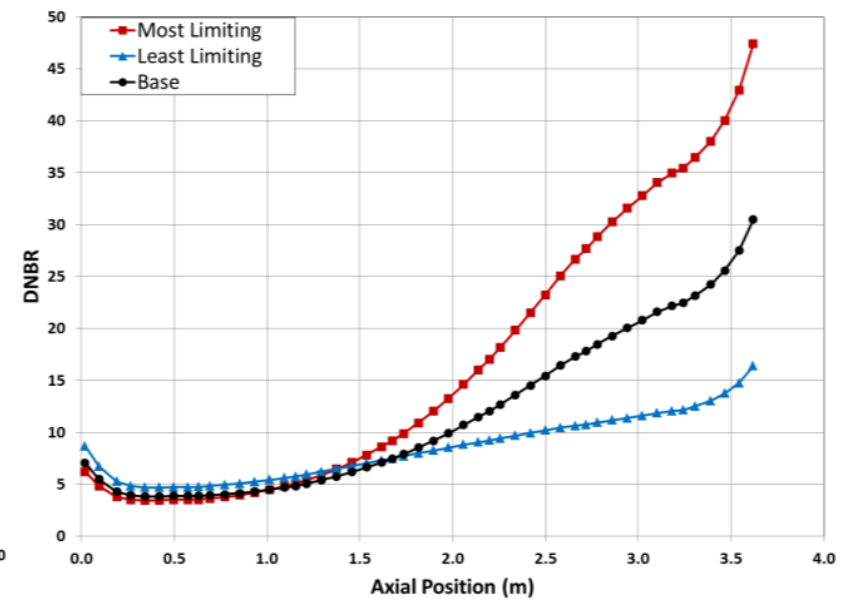

(b)

Figure 9. HF scenario: Heat flux (a) and DNBR (b) as a function of the axial distance from the channel inlet in the channel where the MDNBR event occurred for the most limiting (red squares), base (black circles), and least limiting (blue triangles) cases. 
Figure 12 shows the pin power distribution for an axial slice taken at 0.386 meters from the core inlet for the most limiting MDNBR case. The row-column indexing of the core assemblies shows that the maximum pin power distribution is centered about assembly H-6. Assembly H-6 is the location of the stuck RCCA as shown in Figure 7. Also visible in Figure 12 are the assemblies of lower enrichment where the pin power is less than in higher enriched assemblies. Clearly, the stuck RCCA has a profound effect on the MDNBR in the MSLB accident scenario.

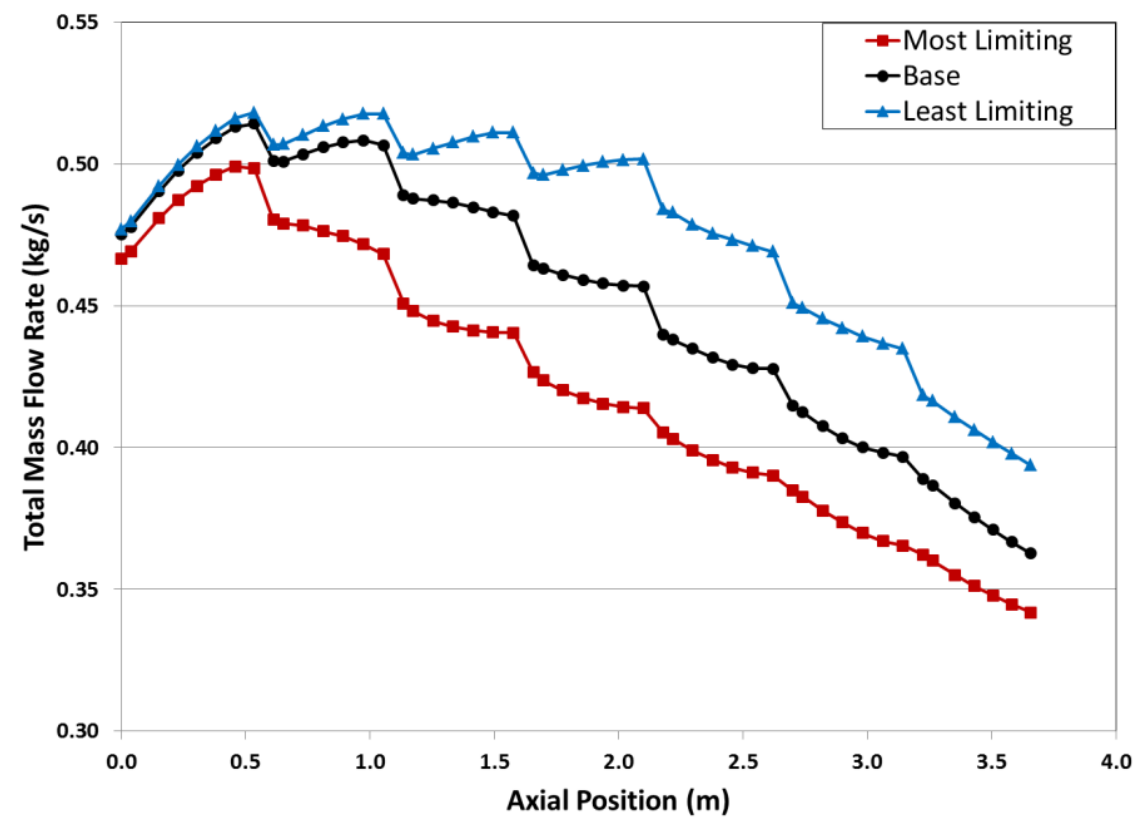

Figure 10. HF scenario: Total mass flow rate as a function of the axial distance from the channel inlet in the channel where the MDNBR event occurred for the most limiting (red squares), base (black circles), and least limiting (blue triangles) cases.

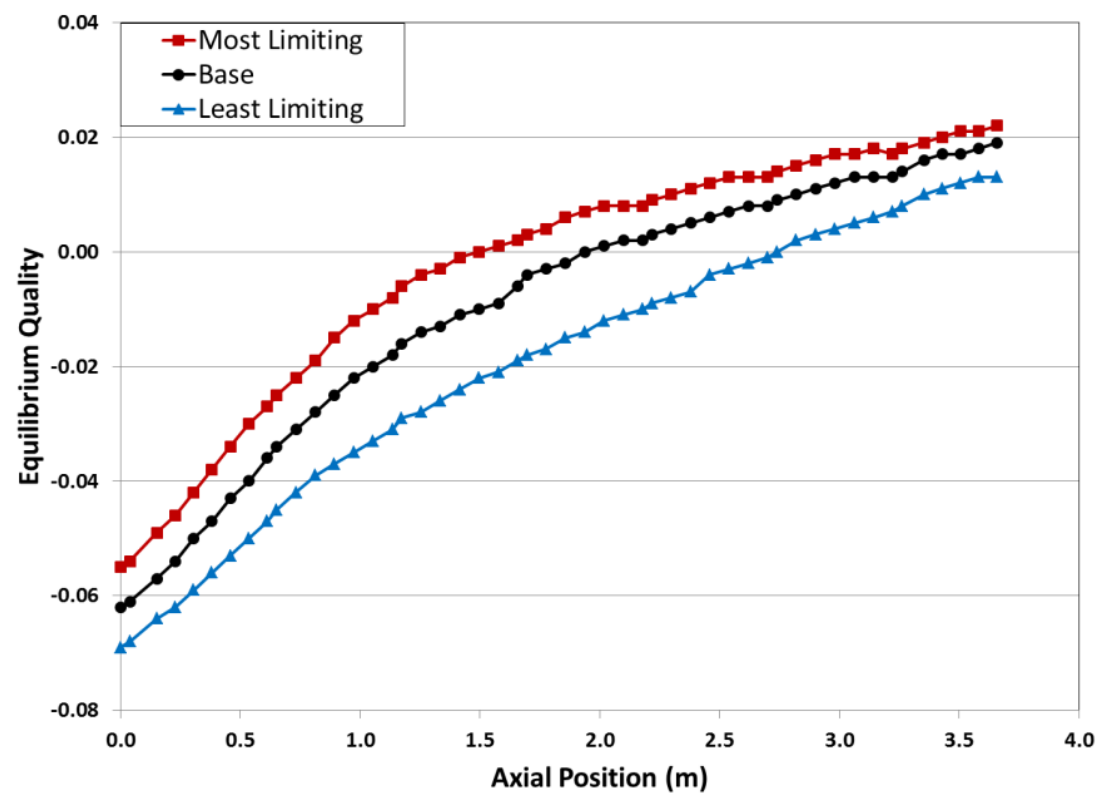

Figure 11. HF scenario: Equilibrium quality as a function of the axial distance from the channel inlet in the channel where the MDNBR event occurred for the most limiting (red squares), base (black circles), and least limiting (blue triangles) cases. 


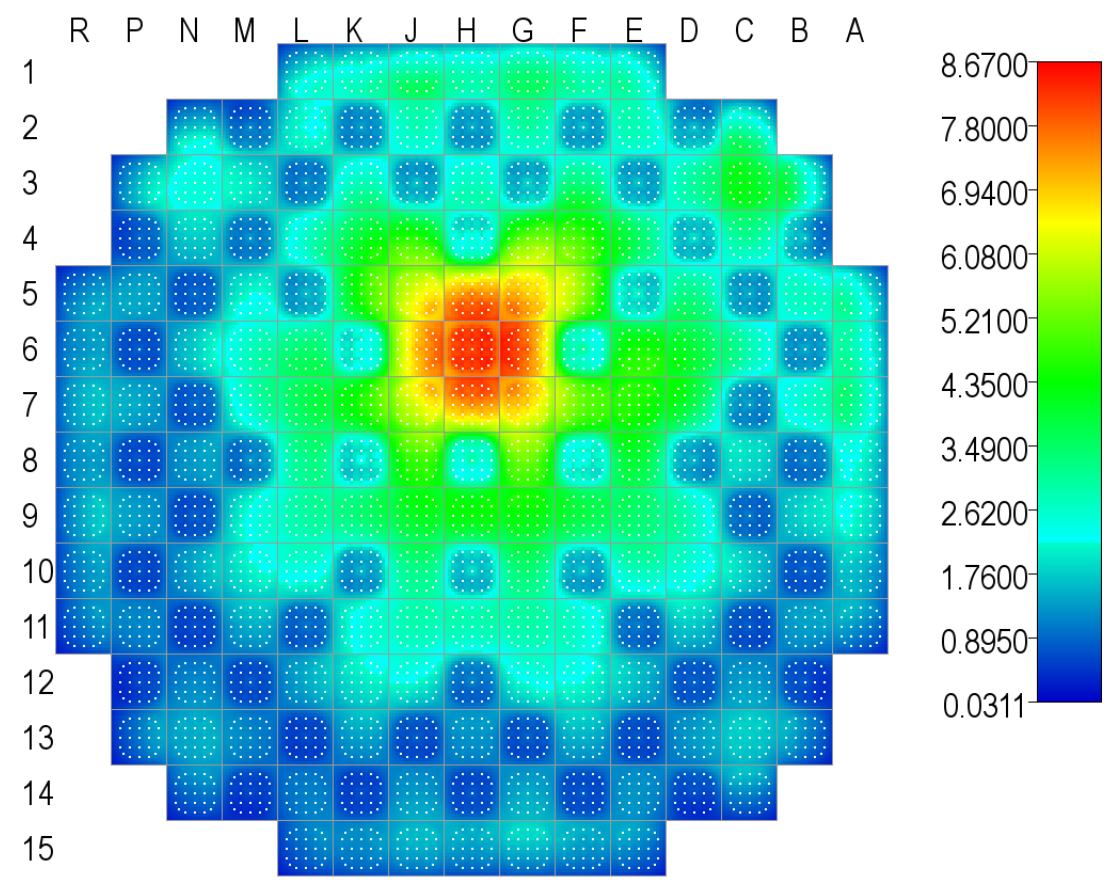

Figure 12. HF scenario: Pin power distribution for an axial slice taken $0.386 \mathrm{~m}$ from the core inlet. Image was produced using the VERAView software developed at ORNL.

\subsection{Low Flow Scenario}

The LF scenario VERA-CS model results for DNBR and heat flux as functions of distance from the core inlet are shown in Figure 13 for the most limiting, base, and least limiting cases in the MDNBR channel. As in the HF scenario, the MDNBR occurred in the assembly with the stuck RCCA (assembly K-8) although the MDNBR occurred lower axially in the LF scenario at around 0.30 meters for each case. The axial power shape peak is again observed in the lower region of the core and the MDNBR is an inverse function of axial power shape. However, the MDNBR values along the flow channel were significantly higher than those in the HF scenario. The effects of spacer grids are less visible in the axial power shape of the LF scenario but are still observed in the total coolant mass flow rate (Figure 14). The axial coolant mass flow rate distributions are distinctly different for the HF and LF scenarios. The coolant mass flow rate distribution of the LF scenario is an effect of the crossflow due to a large radial power gradient in the reactor core. As with the HF case, changes in mass flow rate occur in the hot channel due to cross flow effects between subchannels but total mass conservation is observed when considering the entire reactor core. Similar mass flow change effects were observed in previous studies using different neutronic and thermal-hydraulic code system (Morita, et al. 1995). The equilibrium quality distribution in the DNB limiting subchannel is shown in Figure 15. In the LF scenario, liquid superheat occurs at a lower axial elevation and the equilibrium quality then flattens out as axial elevation increases. This indicates some equilibrium behavior between the vapor and liquid mass fractions where, at the lower inlet mass flow rate, buoyancy effects become substantial and the total mass flow rate follows a flattened shape at higher axial elevations in the channel. Significant voiding in the channel where MDNBR occurred was experienced in this scenario as well, where the exit void fractions were $47.8 \%, 43.6 \%$, and $37.7 \%$ for the most limiting, base, and least limiting cases, respectively. 


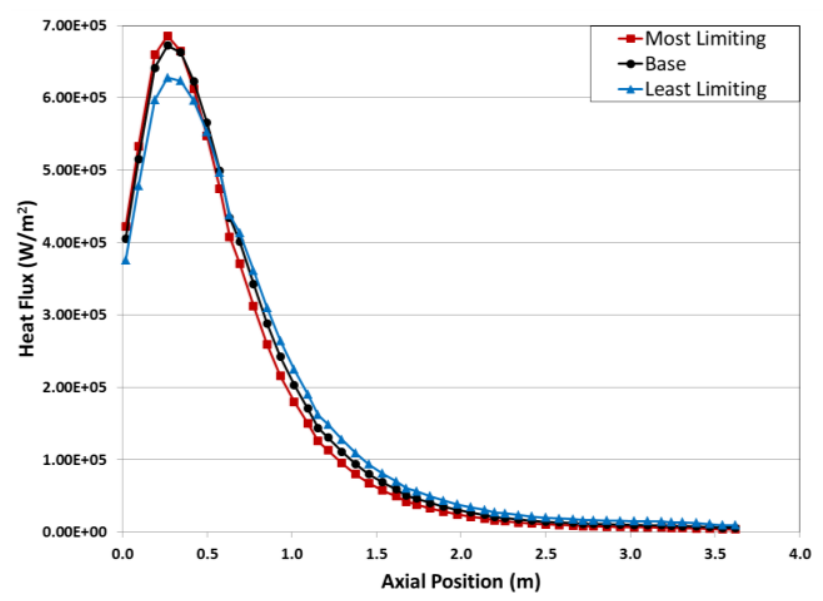

(a)

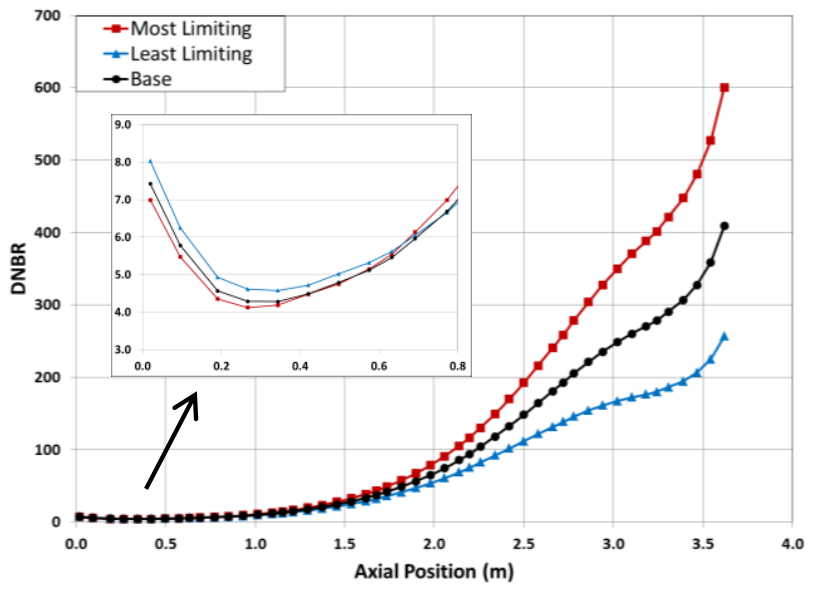

(b)

Figure 13. LF scenario: Heat flux (a) and DNBR (b) as a function of the axial distance from the channel inlet in the channel where the MDNBR event occurred for the most limiting (red squares), base (black circles), and least limiting (blue triangles) cases. The MDNBR data (b) also shows an inset zoomed to the location where MDNBR occurred.

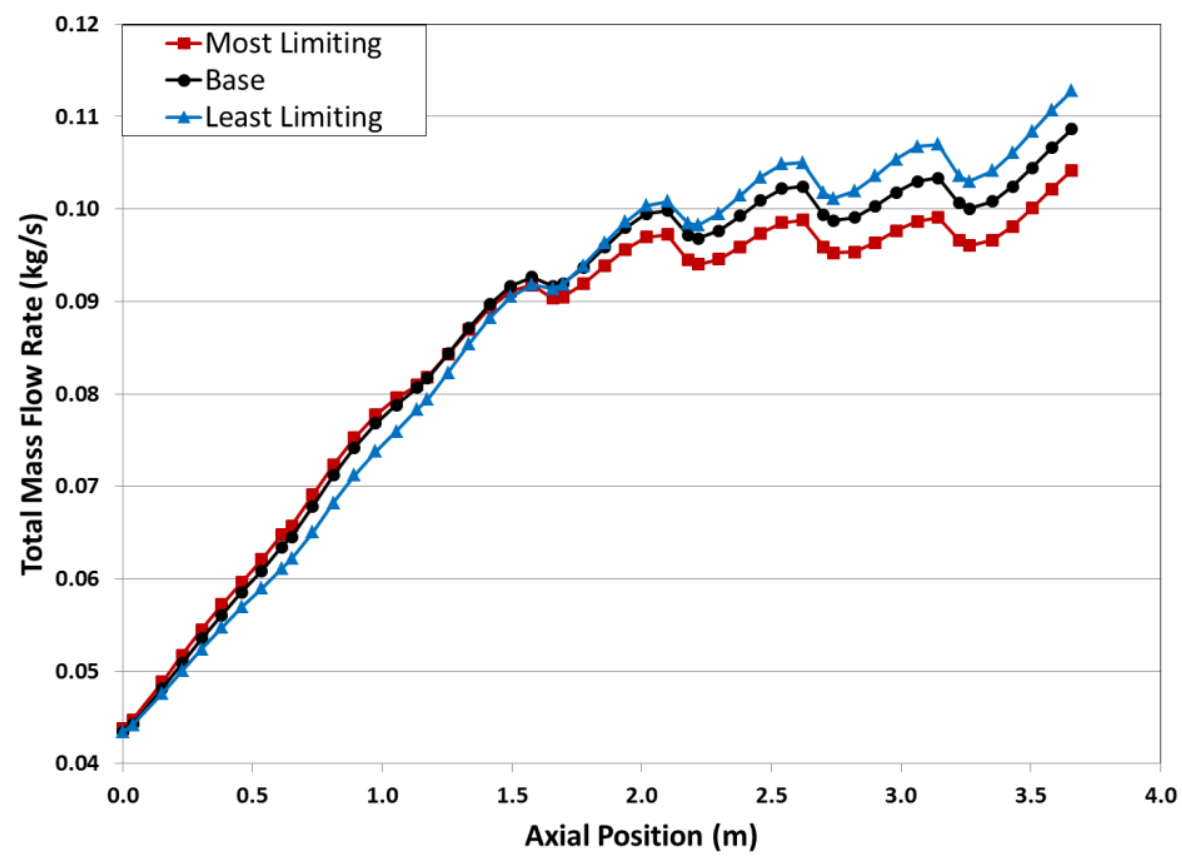

Figure 14. LF scenario: Total mass flow rate as a function of the axial distance from the channel inlet in the channel where the MDNBR event occurred for the most limiting (red squares), base (black circles), and least limiting (blue triangles) cases. 


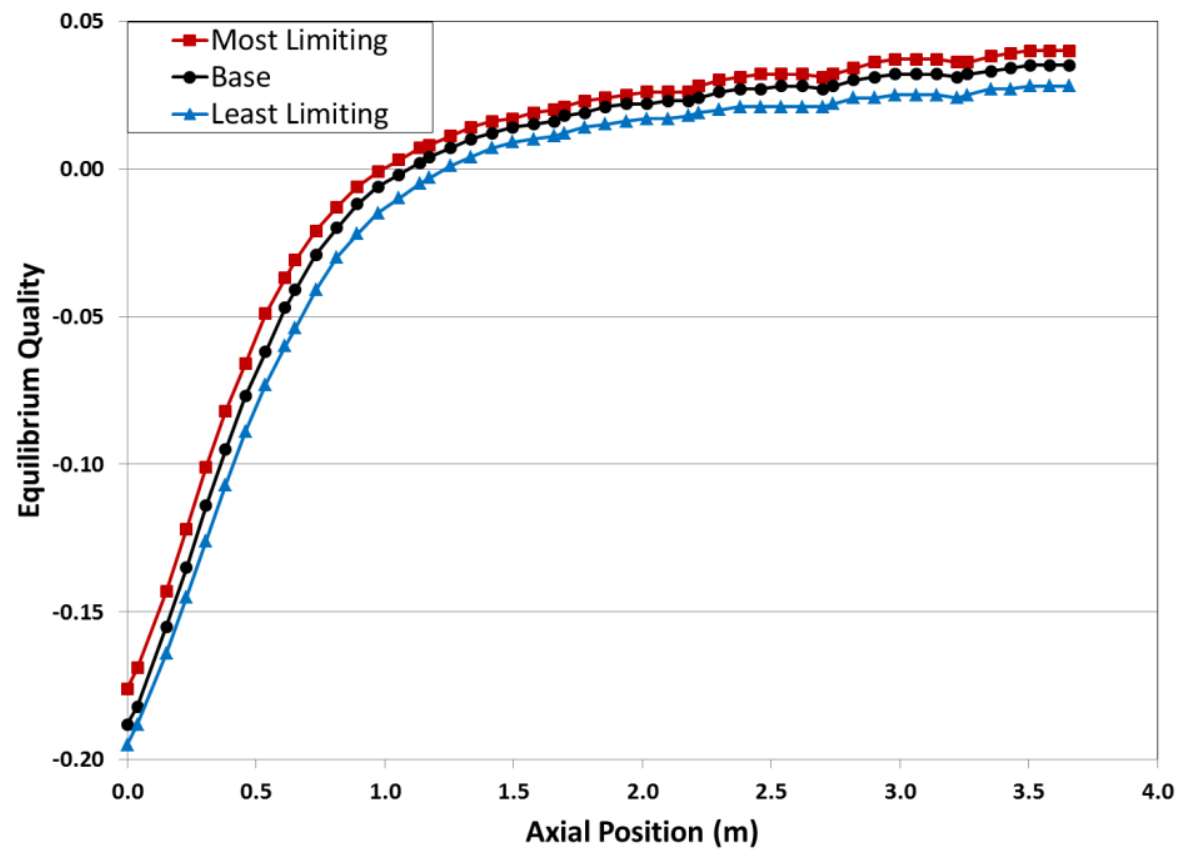

Figure 15. LF scenario: Equilibrium quality as a function of the axial distance from the channel inlet in the channel where the MDNBR event occurred for the most limiting (red squares), base (black circles), and least limiting (blue triangles) cases.

\subsection{Wilks' Nonparametric Approach}

The MDNBR location and value were found for each of the 59 cases in both HF and LF scenarios using the VUSAT as described in Section 3.4. The MDNBR values were then ranked from lowest (most limiting) to highest (least limiting). Wilks' nonparametric statistical approach dictates that by randomly sampling the judiciously chosen input parameters within the space defined by the uncertainty ranges, the 95/95 (95\% probability with $95 \%$ confidence level) tolerance limit can be found. The MDNBR value for the most limiting case was 3.42 with a standard error of \pm 0.08 in the HF scenario and 4.12 with a standard error of \pm 0.04 in the LF scenario. The standard error was calculated at the $95^{\text {th }}$ percentile by the method of Harding, et al. (2014).

\subsection{Goodness-of-Fit Test}

Liang et al. (2011) have applied a parametric approach to determine the 95/95 tolerance limit of peak clad temperature in a LOCA event using 59 samples. If the outcome distribution of the UQ cases can be identified as a certain distribution, such as normal or uniform, by a goodness-of-fit test then population mean $\left(\mu_{p}\right)$ and population standard deviation $\left(\sigma_{p}\right)$ can then be projected by the sample mean $\left(\mu_{s}\right)$ and sample standard deviation $\left(\sigma_{s}\right)$ under a certain confidence level (Liang, et al. 2011), such as the 95\% confidence level of interest in nuclear reactor licensing. The sample mean $\left(\mu_{s}\right)$ and sample standard deviation $\left(\sigma_{s}\right)$ are defined as:

$$
\mu_{s}=\sum_{i=1}^{N} \frac{x_{i}}{N}, \quad \sigma_{s}=\sqrt{\frac{\sum_{i=1}^{N} x_{i}^{2}}{N-1}-\left(\frac{N}{N-1}\right) \mu_{s}^{2}}
$$


where $\mathrm{N}$ is again the number of samples. If the normal distribution can be assumed by the goodness-of-fit test then the population mean $\left(\mu_{p}\right)$ and population standard deviation $\left(\sigma_{p}\right)$ can be calculated for a given confidence level (Liang, et al. 2011):

$$
\mu_{p} \leq\left[\mu_{s}+t_{\alpha}(N-1) \frac{\sigma_{s}}{\sqrt{N}}\right], \quad \sigma_{p}^{2} \leq \frac{\sigma_{s}^{2}(N-1)}{\chi_{1-\alpha}^{2}(N-1)}
$$

where $t_{\alpha}(N-1)$ and $\chi_{1-\alpha}^{2}(N-1)$ are the student $t$ and $\chi^{2}$ variables at $(1-\alpha)$ confidence level with $(N-1)$ degrees of freedom, respectively. Once the population mean and population standard deviation are projected at the $95 \%$ confidence level $\left(\mu_{p, 95 \%}, \sigma_{p, 95 \%}\right)$ the $95 / 95$ coverage is then (Liang, et al. 2011):

$$
Y_{95 / 95}=\mu_{p, 95 \%}-1.645 \sigma_{p, 95 \%}
$$

where, in this work, the second term in Eq. (5) is subtracted from the population mean since lower values of the selected FOM are more limiting. The Pearson Chi-square test (Devore 2004) was used and found that the goodness-of-fit was acceptable for a normal distribution under the $95 \%$ confidence level for both the HF and LF cases. The population mean and standard deviation for MDNBR were then calculated for the HF scenario using Eq. (4) and were found to be 3.99 and 0.029 , respectively. The population mean and standard deviation for MDNBR in the LF scenario were found to be 4.36 and $9.49 \times 10^{-4}$, respectively. Using Eq. (5), the most limiting values of MDNBR with this parametric approach are 3.76 in the HF scenario and 4.31 in the LF scenario.

Table 5 shows that MDNBR values calculated with both the Wilks' and parametric approaches are well above the safety criteria of 1.45 associated with the W-3 correlation at the low pressure condition (Sherder and McHugh 1998) and the Wilks' approach consistently gives the more conservative value. The BEPU results indicate that fuel integrity is maintained for this representative PWR core in a MSLB accident scenario with the most reactive RCCA stuck out of the core with or without continued reactor coolant pump operation.

Table 5. MDNBR values for HF and LF scenarios.

\begin{tabular}{|c|c|c|}
\hline MDNBR Calculation Approach & High Flow & Low Flow \\
\hline Wilks' & 3.42 & 4.12 \\
\hline Parametric & 3.76 & 4.31 \\
\hline
\end{tabular}

\subsection{Sensitivity Analysis}

Sensitivity analysis (SA) seeks to determine the contribution of the uncertainty in specific inputs to the uncertainty in analysis results (Helton, et al. 2006). SA indicates which model inputs have the largest influence on model results and where future work should be dedicated to eliminating epistemic uncertainty. Comprehensive Monte Carlo based global sensitivity analyses use large sample sizes to determine the trends between model inputs and outputs as well as qualitative measures such as correlation coefficients that provide the degree of linearity that exists between inputs and outputs. Correlation coefficients are valued between -1 and +1 where -1 represents a perfect inversely correlated linear relationship and +1 represents a perfect linear relationship. A value close to 0 indicates that the input has insignificant effect on the output. Absolute values of the correlation coefficients between model inputs and a particular FOM can then be ranked from those inputs that are the most influential to those that are the least influential on the FOM. The Pearson, or sample, correlation coefficient (CC) (Helton, et al. 2006) is often examined in sensitivity analysis (Ikonen 2016). Although this work was limited to 59 samples per case for UQ due to the significant computational demand, correlation coefficients can still be calculated to show the trends in which model inputs have the greatest influence on the output uncertainty. 
The CC between MDNBR and each of the perturbed inputs was calculated in Microsoft Excel using the PEARSON function.

Sensitivity analysis results are shown in Table 6 for the HF scenario. The inputs to the W-3 correlation (coolant mass flow rate, coolant temperature, system pressure) are all influential on the MDNBR. The pellet-to-clad gap heat transfer coefficient is the second most influential parameter and more quantitative sensitivity analysis studies may indicate that fuel performance calculations are needed to supplement the full core simulations. The $\beta_{\mathrm{sp}}$ parameter is ranked as the sixth most influential parameter for the MSLB simulations. In situations where high voiding takes place such as this, where the void fraction is almost as much as $50 \%$ at the channel exit in the most limiting case, the two-phase flow approximations begin to have an effect on the results and simulations would benefit from more physically based two-phase flow models. The CCs here show that fuel specifications are the least influential parameters. However, the SA results shown here are certainly not comprehensive although a good indication of those parameters that are highly influential on MDNBR.

Table 6. Pearson correlation coefficients and importance rank for each input parameter (HF scenario).

\begin{tabular}{|c|c|c|}
\hline Model Input Parameter & Pearson Correlation Coefficient (CC) & Rank \\
\hline System pressure & 0.6836 & 1 \\
\hline $\begin{array}{l}\text { Pellet-to-clad gap heat transfer } \\
\text { coefficient }\end{array}$ & -0.6239 & 2 \\
\hline Inlet coolant temperature & -0.4673 & 3 \\
\hline Core power & -0.4509 & 4 \\
\hline Inlet coolant mass flow rate & 0.2395 & 5 \\
\hline $\begin{array}{c}\beta_{\mathrm{sp}}: \text { CTF constant turbulent mixing } \\
\text { coefficient }\end{array}$ & -0.1447 & 6 \\
\hline Grid spacer form loss - MID & -0.1349 & 7 \\
\hline Grid spacer form loss - END & -0.1349 & 8 \\
\hline Fuel $\%$ of theoretical density & 0.1274 & 9 \\
\hline $\begin{array}{c}K_{m}: \text { distribution weighting factor for } \\
\text { the CTF void-drift model }\end{array}$ & 0.1086 & 10 \\
\hline $\begin{array}{l}\text { dhfrac: Fraction of power deposited } \\
\text { directly in coolant }\end{array}$ & 0.0723 & 11 \\
\hline Clad inner radius & 0.0722 & 12 \\
\hline Fuel pellet radius & 0.0653 & 13 \\
\hline Clad outer radius & -0.0622 & 14 \\
\hline Fuel enrichment & 0.0250 & 15 \\
\hline
\end{tabular}

Sensitivity analysis results for the LF scenario are shown in Table 7 with some distinct differences from the HF scenario. The core power is ranked as the most influential parameter on MDNBR with a CC value nearing a perfectly inversely correlated linear relationship. The pellet-to-clad gap heat transfer coefficient is again the second most influential parameter. The inputs to the W-3 correlation are again influential on MDNBR although the dhfrac (gamma heating) parameter has significant importance in this case. The remaining parameters, such as fuel specifications, begin to have negligible $\mathrm{CC}$ values although it is interesting there is some sign change in CCs between the two cases (e.g. clad inner/outer radii). Interestingly, the $\beta_{\text {sp }}$ parameter is ranked as least influential in the LF scenario although significant voiding is experienced. More detailed SA methods, such as Monte Carlo methods that reduced the computational overhead (Wu, Zhang and Abdel-Khalik 2012), are needed to adequately determine the sensitivity of MDNBR to the perturbed input parameters although the results presented here reflect some expected trends. 
Table 7. Pearson correlation coefficients and importance rank for each input parameter (LF scenario).

\begin{tabular}{|c|c|c|}
\hline Model Input Parameter & Pearson Correlation Coefficient (CC) & Rank \\
\hline Core power & -0.9426 & 2 \\
\hline $\begin{array}{c}\text { Pellet-to-clad gap heat transfer } \\
\text { coefficient }\end{array}$ & -0.5228 & 3 \\
\hline System pressure & 0.3333 & 4 \\
\hline $\begin{array}{c}\text { Inlet coolant temperature } \\
\text { dhfrac: Fraction of power deposited } \\
\text { directly in coolant }\end{array}$ & -0.1452 & 5 \\
\hline Inlet coolant mass flow rate & -0.1354 & 6 \\
\hline Grid spacer form loss - END & 0.1186 & 7 \\
\hline Grid spacer form loss - MID & -0.1165 & 8 \\
\hline Fuel enrichment & -0.1165 & 9 \\
\hline Fuel pellet radius & 0.0852 & 10 \\
\hline Clad inner radius & 0.0850 & 11 \\
\hline $\begin{array}{c}K_{m}: \text { distribution weighting factor for } \\
\text { the CTF void-drift model }\end{array}$ & -0.0810 & 12 \\
\hline Fuel \% of theoretical density & 0.0578 & 13 \\
\hline Clad outer radius & -0.0531 & 14 \\
\hline$\beta_{\text {sp: }: \text { CTF constant turbulent mixing }}$ & 0.0463 & 15 \\
\hline
\end{tabular}

\section{CONCLUSIONS}

A new BEPU analysis was demonstrated using the Wilks' nonparametric statistical approach and a parametric approach based on goodness-of-fit of the 4-loop reactor core responses to two MSLB accident scenarios. The CASL nuclear reactor core analysis code VERA-CS was used to simulate the full core response to a MSLB event for 59 cases with perturbed input variables so that the MDNBR value could be obtained at the 95/95 tolerance level. A toolkit named VUSAT was used to perform the UQ studies in an automated fashion. The results show that this typical PWR core retains design margin with respect to the MDNBR safety limit during a MSLB accident with the most reactive RCCA stuck out of the core with or without continued reactor coolant pump operation, as the calculated W-3 MDNBR is significantly above the limit. The HF scenario, where reactor coolant pumps continue to operate, was shown to have the more limiting values of MDNBR as compared to the LF scenario. Fully-coupled neutronic and thermalhydraulic calculations with VERA-CS reduce the input uncertainty in the reactor power factors. The BEPU application shows that uncertainty quantification could identify available margin in the reactor core response with respect to DNB in MSLB accident scenarios. Correlation coefficients between model inputs and outputs show the trends for which input parameters are most influential on MDNBR. Future work will focus on coupling fuel performance calculations for UQ as well as performing more quantitative SA studies.

\section{ACKNOWLEDGEMENTS}

This research made use of the resources of the High Performance Computing Center at Idaho National Laboratory, which is supported by the Office of Nuclear Energy of the U.S. Department of Energy under Contract No. DE-AC07-05ID14517. The authors would like to thank Andrew Godfrey, Mark Baird and Robert Salko at ORNL for their assistance with VERA-CS and Andy Detar, William Higby, Yiban Xu and Liping Cao of Westinghouse for their assistance in providing the system transient condition and the core inlet distributions. This manuscript has been authored by Battelle Energy Alliance, LLC under Contract No. DE-AC07-05ID14517 with the U.S. Department of Energy. The United States Government 
retains and the publisher, by accepting the article for publication, acknowledges that the United States Government retains a nonexclusive, paid-up, irrevocable, world-wide license to publish or reproduce the published form of this manuscript, or allow others to do so, for United States Government purposes.

\section{REFERENCES}

Boyack, B. E. , et al. "Quantifying reactor safety margins part 1: An overview of the code scaling, applicability, and uncertainty evaluation methodology " Nuclear Engineering and Design 119 (1990): 1-15.

Brown, C.S., and Hongbin Zhang. "Uncertainty quantification and sensitivity analysis with CASL Core Simulator VERA-CS." Annals of Nuclear Energy 95 (2016): 188-201.

Collins, Benjamin, and Andrew Godfrey. "Analysis of the BEAVRS benchmark using VERA-CS." ANS MC2015 - Joint International Conference on Mathematics and Computation (M\&C), Supercomputing in Nuclear Applications (SNA), and the Monte Carlo (MC) Method (American Nuclear Society, LaGrange Park, IL (2015)), April 19-23 2015.

Collins, Benjamin, S. Stimpson, B. Kochunas, T. Downar, and W. Martin. "Assessment of 2D/1D capability in MPACT." PHYSOR 2014, Sept. 28 - Oct. 32014.

Devore, J. L. . Probability and Statistics for Engineering and Sciences. The Thompson Corporation, 2004.

Franceshini, F. , et al. "AP1000 PWR Startup core modeling and simulation with VERA-CS." Advances in Nuclear Fuel Management V (ANFM 2015) (American Nuclear Society, LaGrange Park, IL), March 29 - April 12015.

Frepoli, Cesare. "An overview of Westinghouse realistic large break LOCA Evaluation Model." Science and Technology of Nuclear Installations 2008 (2007).

Gauld, I. C. , G. Radulescu, G. Ilas, B. D. Murphy, M. L. Williams, and D. Wiarda. "Isotopic depletion and decay methods and analysis capabilities in SCALE." Nuclear Technology 174, no. 2 (2011): 169.

Godfrey, Andrew, et al. VERA benchmarking results for Watts Bar Nuclear Plant Unit 1 cycles 1-12. CASL Report CASL-U-2015-0206-000, 2015.

Harding, B. , C. Tremblay, and D. Cousineau. "Standard errors: A review and evaluation of standard error estimators using Monte Carlo simulations." The Quantitative Methods For Psychology 10, no. 2 (2014).

Helton, J. C. , J. D. Johnson, C. J. Sallaberry, and C. B. Storlie. "Survey of sampling-based methods for uncertainty and sensitivity analysis." SANDIA Report SAND2006-2901, June 2006.

Huegel, D. S., et al. . RETRAN-02 Modeling and Qualification for Westinghouse Pressurized Water Reactor Non-LOCA Safety Analysis. WCAP-15234-A, Westinghouse Electric Company, 1999.

Ikonen, Timo. "Comparison of global sensitivity analysis methods - Application to fuel behavior modeling." Nuclear Engineering and Design 297 (2016): 72-80.

Ivanov, Kostadin N. , Tara M. Beam, Anthony J. Baratta, Adi Irani, and Nick Trikouros. Pressurised Water Reactor Main Steam Line Break (MSLB) Benchmark. US Nuclear Regulatory Comission, OECD Nuclear Energy Agency, 1999.

Kochunas, Bendan, et al. "VERA core simulator methodology for PWR cycle depletion." ANS MC2015 Joint International Conference on Mathematics and Computation $(M \& C)$, Supercomputing in Nuclear Applications (SNA), and the Monte Carlo (MC) Method (American Nuclear Society, La Grange Park, IL (2015)), April 19-23 2015.

Kucukboyaci, V., Y. Sung, Y. Xu, L. Cao, and R. K. Salko. "VERA-CS Modeling and simulation of PWR main steam line break core response to DNB." ICONE24-60865, International Conference on Nuclear Engineering, June 26-30 2016.

Liang, Thomas K. S. , Ling-Yao Chou, Zhongwei Zhang, Hsiang-Yu Hsueh, and Min Lee. "Development and application of a deterministic-realistic hybrid methodology for LOCA licensing anlysis." Nuclear Engineering and Design 241 (2011): 1857-1863. 
Marcum, W. R., and A. J. Brigantic. "Applying uncertainty and sensitivity on thermal hydraulic subchannel analysis for the multi-application small light water reactor." Nuclear Engineering and Design 293 (2015): 272-291.

Martin, Robert P. , and Larry D. O'Dell. "AREVA's realistic large break LOCA analysis methodology." Nuclear Engineering and Design 235 (2005): 1713-1725.

Morita, T. , et al. "Subchannel Thermal-Hydraulic Analysis at AP600 Low-Flow Steam-Line-Break Conditions." Nuclear Technology 112, no. 3 (1995): 401-411.

Perez, M. , et al. "Uncertainty and sensitivity analysis of a LBLOCA in a PWR Nuclear Power Plant: Results of the Phase V of the BEMUSE programme." Nuclear Engineering and Design 241 (2011): 4206-4222.

Salko, Robert K. , et al. "Development of COBRA-TF for modeling full-core reactor operating cycles." Advances in Nuclear Fuel Management V (ANFM 2015) (American Nuclear Society, LaGrange Park, IL (2015)), March 29 - April 12015.

Sherder, W.J., and C.J. McHugh. Reactor core response to excessive secondary steam releases. WCAP9227-P-A Revision 1, Westinghouse Electric Company, 1998.

Sung, Y. , V. Kucukboyaci, L. Cao, and R. K. Salko. "COBRA-TF Evaluation and application for PWR steamline break DNB analysis." NURETH16-13431, International Topical Meeting on Nuclear Reactor Thermal Hydraulics, August 30 - September 4 2015a.

Sung, Yixing, Vefa Kucukboyaci, Yiban Xu, and Robert K. Salko. VERA-CS modeling and simulation of PWR MSLB core response to DNB - high flow case. CASL Report L3.VMA.AMA.P11.01, $2015 b$.

Tong, L. S. . "Prediction of departure from nucleate boiling for an axially non-uniform heat flux distribution." Journal of Nuclear Energy 21 (1967): 241-248.

Wilks, S. S. . "Determination of sample sizes for setting tolerance limits." The Annals of Mathematical Statistics 12, no. 1 (1941): 91-96.

Wu, Zeyun, Qiong Zhang, and Hany Abdel-Khalik. "Hybrid Monte Carlo-Deterministic Methods for Reactor Analysis." Nuclear Technology 180, no. 3 (December 2012): 372-382.

Zhang, Hongbin, Ronaldo Szilard, Paul Bayless, Ling Zou, and Haihua Zhao. "Early-demonstration of BEPU analysis with the RELAP5-3D code in response to proposed 10 CFR 50.46(c) rulemaking." 2016 International Congress on Advances in Nuclear Power Plants (ICAPP 2016), April 17-20 2016. 